\title{
EQUIDIFFERENÇA E PROPORÇÃO: QUAL A RELAÇÃO DESTES CONTEÚDOS COM O ENSINO DE ÁLGEBRA NA INSTRUÇÃO ELEMENTAR DO FINAL DO SÉCULO XIX E INÍCIO DO SÉCULO XX?
}

\author{
Jeremias Stein Rodriguês* \\ David Antonio da Costa**
}

\section{RESUMO}

Este texto problematiza relações do ensino de equidifferença e proporção com outros conteúdos no ensino elementar. A partir de base teórico metodológica da história cultural foram analisados livros didáticos do final do século XIX e início do século XX e dois relatórios estadunidenses. Observou-se a relação destes conteúdos com o tema de progressões e saberes algébricos, sem que estes se estabelecessem como uma preparação para a abordagem da Álgebra no ensino secundário.

Palavras-chave: ensino de álgebra, história da educação matemática, resolução de equações, livro didático.

* Instituto Federal de Santa Catarina (IFSC), Florianópolis/SC, Brasil.

${ }^{* *}$ Universidade Federal de Santa Catarina (UFSC), Florianópolis/SC, Brasil. 
EQUIDIFERENCIA Y PROPORCIÓN: ¿CUÁL ES LA

RELACIÓN DE ESTOS CONTENIDOS CON LA

ENSEÑANZA DEL ÁLGEBRA EN LA INSTRUCCIÓN

PRIMARIA DEL FINAL DEL SIGLO XIX Y EL INICIO DEL

SIGLO XX?

\section{RESUMEN}

Este texto problematiza las relaciones entre la enseñanza de la equidifferencia y la proporción con otros contenidos en educación primaria. Com la base teórica y metodológica de la historia cultural, se analizaron libros de texto de finales del siglo XIX y principios del siglo XX y de los informes estadounidenses. Se observó la relación de estos contenidos con el tema de las progresiones y el conocimiento algebraico, sin que estos se establecen como una preparación para el enfoque de la álgebra en la educación secundaria.

Palabras clave: enseñanza de álgebra, historia de la educación matemática, resolución de ecuaciones, libro didáctico.

\section{EQUIDIFFERENCE AND PROPORTION: WHAT \\ ISTHERELATIONOF THESE CONTENTS TO THE \\ TEACHING OF ALGEBRA IN ELEMENTARY EDUCATION \\ IN THE END OF THE $19^{\text {TH }}$ CENTURY AND BEGINING OF \\ THE 20 ${ }^{\text {TH }}$ CENTURY?}

\section{ABSTRACT}

This text problematizes the relations between the teaching of equidifference and proportion with other contents of the elementary education. From the theoretical and methodological basis of cultural history, textbooks from the late 19th and early 2oth centuries and two American reports were analyzed. The relation of these contents with progressions and algebraic knowledge was observed, without these being established as a preparation for the approach to Algebra in secondary education.

Keywords: algebra teaching, history of mathematics education, equation's solving, textbook.

\section{ÉQUIDIFFÉRENCE ET PROPORTION: QUELLE EST LA \\ RELATION DE CES CONTENUS AVEC \\ L'ENSEIGNEMENT DE L'ALGÈBRE DANS \\ L'INSTRUCTION ÉLÉMENTAIRE DE LA FIN DU XIXe. SIÈCLE ET DU DÉBUT DU XXe. SIÈCLE?}

\section{RÉSUMÉ}

Ce texte problématise la relation entre l'enseignement de l'équidiférence et la proportion avec 
les autres contenus de l'enseignement élémentaire. Les manuels scolaires de la fin du XIXe siècle et du début du XXe siècle, ainsi que deux rapports américains, ont été analyses d'un point de vue théorique et méthodologique de l'histoire culturelle. On peut voir la relation de ces contenus avec le thème des progressions et des connaissances algébriques, sans ces s'imposer comme une préparation pour approche l'Algèbre dans l'éducation secondaire.

Mots-clés: enseignement de l'Algèbre, histoire de l'enseignement des mathématiques, résolution d'équations, manuel scolaire. 


\section{INTRODUÇÃO}

A ideia de razão entre dois números é, em geral, uma ideia que perpassa diversos níveis no âmbito do ensino da matemática. Buscando colocar de forma simples, uma razão é uma constante que estabelece uma relação entre dois números. Em geral, podem ser caracterizadas por uma relação de soma/diferença, chamadas também de razões aritméticas; ou de multiplicação/quociente, denominadas de razões geométricas. Deste modo, se forem tomados os números 12 e 3, pode-se exemplificar que uma razão por diferença dos dois é 9 , uma vez que $12-3=9$, o que implica que há 9 unidades entre 3 e 12 , ou seja $3+9=12$. De forma semelhante, uma razão por quociente dos números é 4, representada como 12: $3=4$, indicando assim que é possível, adicionando o número 3 quatro vezes, obtermos o resultado 12 , ou seja $3 \times 4=$ 12.

Disso é possível compreender o que são proporções aritméticas e geométricas. Segundo Euler,

Quando duas razões aritméticas, ou relações, são iguais, esta igualdade é chamada de proporção aritmética.

Portanto, quando $a-b=d$, e $p-q=d$, de modo que a diferença dos números $p$ e $q$ é a mesma que dos números $a$ e $b$, dizemos que estes quatro números formam uma proporção aritmética; para a qual então escrevemos $a-b=p-q$, expressando claramente que a diferença entre $a$ e $b$ é igual à diferença entre $p$ e $q .(1828, p .129$, tradução pelos autores)

Assim, como $12-3=9$ e $15-6=9$, se estabelece a proporção aritmética $12-3=15-6$.De modo semelhante Euler apresenta as proporções geométricas 
Portanto, por exemplo, escrevemos $a: b=c: d$, ou $a: b:: c: d^{1}$, para indicar que a relação $a$ : $b$ é igual a relação $c: d$; mas isto é mais facilmente explicado dizendo que $a$ está para $b$ assim como $c$ está para $d(1828$, p. 152, tradução pelos autores).

Do fato que $12: 3=4$ e que $28: 7=4$, fica então caracterizada a proporção geométrica 12:3 $=28: 7$, ou ainda, utilizando a notação da época, $12: 3:: 28: 7$. Igualmente a proporção aritmética vista, $12-3=15-6$, pode ser representada como $12.3: 15.6$.

A partir de um estudo histórico que buscou discutir a origem dos termos progressão, ou proporção, aritmética/geométrica, Cojori (1922) aponta que certos autores teriam utilizado o termo "proporção" sem designar se estas seriam "aritmética" ou "geométrica". No Brasil, um movimento semelhante ocorre, uma vez que uma análise sobre alguns livros do ensino de aritmética apresenta que, em títulos como Proporções (TRAJANO, 1922, p. 99) ou Razões e proporções (L. L., 1916, p. 87; SILVA, 1923, p. 39), e, como destaca Silva (1923, p. 39-40, grifos do autor), as razões podem ser

[...] por differença ou por quociente. [...] Se as razões forem por differença a proporção será por differença ou arithmetica ou ainda equidifferença. Se as razões forem por quociente a proporção será por quociente ou geometrica ou proporção propriamente dita.

Ou ainda, nas "razões por differença, a proporção toma o nome de equidifferença, reservando-se o termo proporção propriamente dita para a razão por quociente" (L. L., 1916, p. 87, grifos do autor). O mesmo pode ser constatado no livro de Trajano (1922), que se refere apenas à proporção quando trata de proporções geométricas. Deste modo, no Brasil, os termos equidifferença e

\footnotetext{
${ }^{1}$ De forma semelhante uma notação própria para equidifferenças é utilizada em alguns livros da época, como Silva (1923), L.L. (1916) ou Souza (1910), na qual se $a-b=c-d$, então, escrevese a equidifferença $a . b: c$. $d$. Vale ressaltar que estas notações não são mais utilizadas.
} 
proporção ${ }^{2}$ foram, ao que parece, utilizados para denotar proporções aritméticas e proporções geométricas, respectivamente.

Após apresentar a ideia de equidifferença e proporção, alguns livros de Aritmética lançam outro destaque para a aplicação deste conceito, sendo este a determinação de um ou mais termo(s) para que a proporção esteja estabelecida. Nesse sentido, Silva (1923) apresenta a ideia para determinar um "termo desconhecido" (p. 40), sem indicar o que é esse elemento. Já Trajano, indica que se pode determinar um termo de uma proporção se os outros três forem conhecidos e segue dizendo que "O termo desconhecido é representado na proporção pela lettra x, e chama-se a incognita da proporção" (1922, p. 100, grifo do autor).

Segundo Chervel (1990), a disciplina escolar é constituída para além da abordagem de um conteúdo, tem também, como elemento de seu "núcleo" (p. 205), os exercícios. Além de tomar parte no processo de aprendizagem, os exercícios também denotam tendências adotadas na história da disciplina escolar (CHERVEL, 1990). Neste caso, é possível notar duas abordagens acerca do tratamento dado ao conteúdo escolar de proporções: uma voltada aos processos aritméticos pela realização de contas, como a verificação de que os dois lados da igualdade em uma proporção apresentam o mesmo resultado; a outra, em que se busca determinar um valor desconhecido, de modo que seja satisfeita a condição de proporcionalidade, assumindo assim uma perspectiva algébrica, mas que ainda tende aos processos aritméticos.

Isto ressalta a existência de saberes que poderiam fazer parte da Álgebra ou da Aritmética, não só pela dupla abordagem observada no ensino de Aritmética, pertencente à instrução elementar3, mas por também ser possível constatar que há livros nas duas áreas com a presença desses conteúdos. Contudo, será que este ensino toma a forma de uma preparação para a Álgebra

\footnotetext{
2 Optou-se aqui por utilizar o termo "proporção" para delimitar a separação entre equidifferença e proporção, e o termo "proporções" para designar ambos os conteúdos.

3 Considera-se, aqui, o ensino elementar como o ensino anterior ao ensino secundário.
} 
presente no ensino secundário? A partir das perspectivas da época, seria o ensino deste suficiente como preparação para esta Álgebra secundarista? Além disso, quais relações deste conteúdo podem ser estabelecidas com os demais da época?

De maneira semelhante ao desenvolvido na pesquisa de Neves e Soares (2019), que buscaram perceber a finalidade do ensino de progressões na instrução secundária do século XIX, no Colégio Pedro II, a partir da análise de livros didáticos de Aritmética e Álgebra, este estudo tem como objetivo responder as questões levantadas anteriormente acerca do ensino de proporções. Assim, objetiva-se compreender quais as relações podem ser observadas entre o ensino de equidifferenças e proporções com outros temas da instrução, bem como a relação deste ensino com os saberes algébricos que nela podem ser observados. Para isto, buscou-se compreender a finalidade do conteúdo de proporções no ensino elementar e das discussões acerca da preparação para o ensino de Álgebra na escola secundária no final do século XIX e início do século XX. Cinco livros de aritmética do início do século XX foram utilizados com fontes da pesquisa, que assume um viés historiográfico. Além destes, os relatórios de duas comissões estadunidenses, a Comissão dos dez ${ }^{4}$ e a Comissão dos quinze 5 , que, segundo Rodriguês e Costa (2019), propõem mudanças para o ensino secundário e elementar do país, respectivamente, fomentaram as discussões acerca da relação entre equidifferença e proporção com a Álgebra, bem como quanto a uma possível preparação para o ensino de Álgebra da instrução secundária.

Este artigo se encontra estruturado da seguinte forma: primeiro é apresentado(s) o referencial teórico metodológico que guia(m) a pesquisa desenvolvida; em seguida são analisadas as relações do ensino de proporções a partir dos livros e dos relatórios estadunidenses, de forma a buscar respostas aos questionamentos levantados; por fim, têm-se as conclusões do estudo e as

\footnotetext{
4 "Committee of ten". Recebeu esse nome por ter dez membros.

5 "Committee of fifteen”. Analogamente, possuía quinze membros.
} 
perspectivas para pesquisas futuras.

\section{REFERENCIAL TEÓRICO-METODOLÓGICO}

Esta pesquisa toma como base as perspectivas da historiografia, na história cultural, com base nas perspectivas de Certeau (2013). Através de uma aproximação semelhante, para Le Goff (1990) os documentos são o resultado de uma sociedade que os constitui a partir de relações de forças da época, sendo assim frutos dos vestígios deixados por uma cultura e por memórias coletivas registradas, que são produzidas pelo cotidiano. Partindo disso, segundo Le Goff (1990, p. 472), pode-se considerar que

\footnotetext{
O documento não é inócuo. É antes de mais nada o resultado de uma montagem, consciente ou inconsciente, da história, da época, da sociedade que o produziram, mas também das épocas sucessivas durante as quais continuou a viver, [...] continuou a ser manipulado, ainda que pelo silêncio. [...] O documento é monumento. Resulta do esforço das sociedades históricas para impor ao futuro - voluntária ou involuntariamente - determinada imagem de si próprias.
}

Segundo Chervel (1990), a questão principal a ser feita no âmbito dos estudos históricos, acerca das disciplinas escolares, é "Por que a escola ensina o que ensina?" (p. 190). Nesse sentido, para Chervel (1990), "a tarefa primeira do historiador das disciplinas escolares é estudar os conteúdos explícitos do ensino disciplinar" (p. 203) e, para isso, "Não podemos, pois, nos basear unicamente nos textos oficiais para descobrir as finalidades do ensino" (p. 190). E, para Schubring (2005), os principais determinantes da realidade do ensino são, em primeiro lugar, os manuais e, em segundo lugar, os professores, sendo que as "pesquisas da educação matemática têm mostrado que a realidade de dia-a-dia do ensino é determinada decisivamente pelos manuais" (p. 9). É por meio desta perspectiva que os livros didáticos, principalmente do início do século XX, são 
tomados aqui como documentos de forma a assumirem o papel de fontes privilegiadas para a pesquisa histórica.

Tais documentos, segundo Choppin (2004, p. 553), "exercem quatro funções essenciais, que podem variar consideravelmente segundo o ambiente sociocultural, a época, as disciplinas, os níveis de ensino, os métodos e as formas de utilização". Tais funções, segundo o autor, são: a função referencial, em que o livro didático reflete o programa de ensino, de forma integral ou como uma interpretação, que "constitui o suporte privilegiado dos conteúdos educativos, o depositário dos conhecimentos, técnicas ou habilidades que um grupo social acredita que seja necessário transmitir às novas gerações" (CHOPPIN, 2004, p. 553); a função instrumental, no qual o livro didático faz uso de certas práticas de aprendizagem; a função ideológica e cultural, em que se busca a aculturação ou doutrinação do estudante da cultura e valores da sociedade; a função documental: em que o livro didático, ao fornecer documentos, pode vir a desenvolver a criticidade do estudante.

Deste modo, com base em Choppin (2004), tomar os livros como documentos de análise possibilita que questões como programa de ensino, práticas de aprendizagem e os propósitos do ensino possam ser investigados. Sendo assim, alguns livros didáticos de Aritmética, encontrados no Repositório de Conteúdos Digitais (RCD - UFSC) ${ }^{6}$, foram utilizados neste estudo: Arithmetica Pratica e Formulario (SILVA, 1923), que, como é apresentado por Barbaresco (2019), foi utilizado no ensino primário da Escola de Aprendizes Artífices de Santa Catarina; Arithmetica Elementar Illustrada (TRAJANO, 1922), uma vez que, segundo Oliveira (2019), Trajano foi autor de livros escolares amplamente difundidos no Brasil; Elementos de arithmetica (L.L., 1916), por ser uma obra voltada ao ensino normal e gymnasios, formando o professor para o ensino elementar; e Arithmetica Elementar (SOUZA, 1910), voltado para as escolas primárias e o primeiro ano do curso normal do

\footnotetext{
${ }^{6}$ Espaço virtual em que são alocadas fontes digitais, como as do Grupo de Pesquisa de História da Educação Matemática (GHEMAT), na comunidade História da Educação Matemática, disponível em: https://repositorio.ufsc.br/handle/123456789/1769.
} 
Amazonas. Os livros didáticos supracitados foram empregados como fontes para a discussão acerca das abordagens de ensino de equidifferença e proporção no final do século XIX e início do século XX.

Além destes, o livro Traité D’Arithmétique: Théorique et Pratique (LEYSSENNE, 1911) também foi utilizado para análise. Apesar de o livro de Leyssenne (1911) não ser brasileiro, ganha destaque uma vez que é possível observar que tal obra circula pelo país por meio dos discursos de Cabrita (1917) e Reis (1918a), apontando a relevância deste para o âmbito educacional brasileiro, bem como para as discussões acerca de uma Álgebra para o ensino elementar7. Outro livro, Elements of Algebra (EULER, 1828), também compôs o corpo de fontes desta pesquisa por se tratar de uma obra de referência ou livro elementar que, na visão de Schubring (2003), "visa ao projeto de tornar elementar o saber, de fazê-lo ensinável, sem privilegiar um determinado nível de ensino" (p. 4), de forma a contribuir para as discussões aqui levantadas. É necessário destacar, contudo, que a obra de Euler (1828), talvez não tenha sido escrita com vista ao ensino elementar ou secundário, mas permite a busca por relações do conteúdo de proporções com outros saberes matemáticos. De modo semelhante, a conferência "Os dois ultimos annos de arithmetica, na escola primaria, segundo a Comissão dos quinze” (REIS, 1918a; REIS, 1918b), que depois constitui o prefácio do livro do autor (REIS, 1919), também corroborou para a investigação.

$7 \mathrm{O}$ autor destina o capítulo X de sua obra para falar dos "Princípios elementares da álgebra, e aplicação de seus princípios na resolução de problemas de aritmética”. 


\title{
AS PROPORÇÕES E AS PROGRESSÕES ARITMÉTICAS E GEOMÉTRICAS
}

\begin{abstract}
Progressões aritméticas e geométricas ${ }^{8}$ estão presentes em antigos registros matemáticos [...]. Séries finitas desses dois tipos podem ser encontradas cerca de dois mil anos antes dos nomes proporções e progressões "aritméticas" e "geométricas" fossem introduzidas (COJORI, 1922, p. 734, tradução pelos autores).
\end{abstract}

É assim que Coroji (1922) inicia seu texto, no qual busca abordar a origem dos termos proporção, ou progressão, Aritmética ou Geométrica. $\mathrm{O}$ autor possibilita questionar uma associação entre proporções e progressões. Antes de qualquer outra discussão, talvez seja necessário determinar exatamente o que são as Progressões Aritméticas e Geométricas, de forma a tentar verificar se existem relações com proporções que a elas possam, talvez, ser associadas.

Uma progressão pode ser definida ${ }^{9}$ como uma sequência de termos ordenados, com uma quantidade finita ou infinita ${ }^{10}$ destes, na qual existe uma constante, chamada de razão, que estabelece uma relação entre um termo e o próximo. Na Progressão Aritmética (PA), a relação entre termos é dada pela soma da razão, assim se na PA $(a, b, \ldots)$, em que $a$ e $b$ são termos consecutivos, se $r$ é a razão da progressão, então, deve-se satisfazer a soma do primeiro termo (a) com a razão deve resultar no segundo termo (b), de modo que $b=a+r$ ou, subtraindo $a$ dos dois lados da igualdade, $b-a=r$. De forma geral, se $a$ e $b$ são

\footnotetext{
8 O conteúdo de Progressões Aritméticas e Progressões Geométricas pode(m) ser observado(s) nos dias de hoje no currículo e nos livros do primeiro ano do ensino médio brasileiro. Já os títulos "Proporções Aritméticas" ou "Proporções Geométricas" não fazem mais parte do ensino escolar brasileiro, de forma explícita, como é possível observar em documentos normativos como Parâmetros Curriculares Nacionais (BRASIL, 1997) ou a Base Nacional Comum Curricular (BRASIL, 2018).

9 Na Matemática, algumas vezes, uma definição pode ser apresentada em diversas formas. As apresentadas aqui foram elaboradas pelos autores deste artigo.

${ }^{10}$ Uma progressão é finita se possuir um número finito de termos. De modo semelhante será infinita se possuir infinitos termos.
} 
termos de uma PA de razão $r$ e de modo que $a$ é o antecessor ${ }^{11}$ de $b$, então é verdade que $b-a=r$. Por exemplo, a sequência $(1,4,7,10,13, \ldots)$ é uma PA infinita, pois é possível observar que existe uma razão estabelecida entre seus termos, $r=3$, uma vez que $1+3=4,4+3=7$, e assim por diante. A razão também pode ser observada pela subtração de um termo com o seu antecessor: $13-10=3,10-7=3,7-4=, 3$ e $4-1=3$.

De modo semelhante, a Progressão Geométrica (PG) se estabelece como uma sequência de termos em que o próximo termo é igual ao anterior multiplicado por uma razão. Assim, dada a PG $(a, b, \ldots)$, sendor a razão da PG, o produto de $a$ com a razão deve ter como resultado $b$, com isso $b=a \times r$ ou, dividindo os dois lados da igualdade por $a, b: a=r$. Por exemplo, a sequência $(5,10,20,40)$ é uma PG finita em que se observa uma razão $r=2$ visto que $5 \times 2=10,10 \times 2=20$, e assim sucessivamente. A razão também pode ser observada pela divisão dos termos:40:20 $=2,20: 10=2$ e 10:5 $=2$.

A partir das definições de proporções aritméticas e geométricas apresentadas e cotejadas com as enunciadas em Euler (1828) na introdução deste texto, é possível perceber que se a sequência $(a, b, c, d, \ldots)$ formar uma PA de razão $r$, então deve-se satisfazer que $d-c=r, c-b=r$ e $b-a=r$. Deste modod $-c=c-b=b-a$, no que é possível observar equidifferenças, ou proporções aritméticas, como $d-c=b-a$ ou $c-b=b-a$. De maneira análoga, se a sequência $(a, b, c, d \ldots)$ forma uma PG de razão $r$, então $d: c=$ $r, c: b=r$ e $b: a=r$ no que se observa que $d: c=c: b=b: a$ no que é possível destacar proporções geométricas como, por exemplo, $c: b=d: c$ ou $c: b=b: a$. Logo, pode-se estabelecer que

Termos em progressão geométrica podem ser considerados como termos que estão em proporção geométrica contínua. Sendo assim, na progressão $1,2,4,8,16$ temos 1:2=2:4 $=4: 8=8: 16$. A forma de

\footnotetext{
${ }^{11} \mathrm{O}$ que significa dizer, em outras palavras, que $a$ é um termo da progressão e que $b$ é o próximo termo da sequência.
} 
expressão utilizada por Euclides repousa nesta perspectiva. Termos que eram ditos [...] estar em "progressão geométrica" ele descrevia como termos "em proporção contínua" (COJORI, 1922, p. 736, tradução pelos autores).

Seguindo a mesma linha de raciocínio, pode-se dizer que uma PA descreve termos que estão em proporção aritmética contínua. Assim, percebe-se uma relação muito próxima entre o ensino de proporções e de progressões. Não é possível encontrar a presença do ensino de progressões nos livros de Trajano (1922), L.L. (1916), Leyssenne (1911) ou Souza (1910), mas, contudo, no livro de Silva (1923, p. 42) é apresentado o tópico Series de razões eguaes. Nele o autor apresenta esta série na forma “ $a: b:: c: d:: e: f:: \cdots$, contudo a notação utilizada pelo autor não torna possível que se estabeleça um relação da série apresentada com uma PG. Isto pode ser observado da seguinte forma: dada a série $a: b:: c: d:: e: f$, que pode ser expresso na forma $a: b=c: d=e: f$, entende-se que os termos que possuem a mesma razão, mas se forem tomados $a=12, b=2, c=30$ e $d=5$ tem-se que $12: 2=6$ e $30: 5=6$ mas que a sequência $(2,12,5,30)$ não forma uma PG uma vez que $2 \times 6=12$ mas $12 \times 6=$ 72 e não $5^{12}$. Silva (1923) exibe também algumas propriedades que são satisfeitas pela série por ele apresentada, mas não utiliza o termo progressão.

Já no livro de Euler (1828) encontra-se a abordagem dos dois tipos de proporções e dos dois tipos de progressão. Vale ressaltar ainda que, na exposição do conteúdo, Euler (1828) apresenta os temas proporção aritmética, PA, proporção geométrica e PG, nesta ordem. Isto denota, principalmente, que o ensino de proporções serviria como base para o de progressões, do último fazer parte do ensino elementar ou de outro nível de ensino, uma vez que a obra de Euler seria um livro elementar13 (SCHUBRING, 2003), ou seja, que busca

\footnotetext{
12 Isso mostra que não são todas as proporções que formam uma PA ou uma PG. No caso da PA isso pode ser observado a partir da equidifferença $7.4:: 5.2$, em que os termos $(2,5,4,7)$ não formam uma PA. Mesmo que a ordem dos termos fosse alterada, como $(2,4,5,7)$, a equidifferança ainda não iria caracterizar uma proporção.

13 Livros denominados "elementos" possuem a característica de apresentar as unidades constituintes de uma ciência. Nesse sentido, "[...] a palavra "elementos" designa as partes
} 
evidenciar os elementos da Álgebra. Outro componente importante a ser destacado, é que o fato do livro de Euler (1828), como seu título bem expõe, tratar dos Elementos de Álgebra, indica assim uma relação das proporções, bem como das progressões, com esta área do conhecimento/ensino. Contudo, como destacam Neves e Soares (2019, p. 316), em relação ao ensino secundário,

\footnotetext{
Entre os anos de 1850 e 1856, as Progressões estavam presentes tanto em Aritmética quanto em Álgebra; nos programas de 1858 até 1892 eram abordadas somente em Aritmética; entre os anos de 1893 e 1898, voltam a serem estudadas simultaneamente em Aritmética e Álgebra. De 1899 até 1912, eram vistas somente no campo da Aritmética; [...] a partir do ano de 1915, [...] se consolidam em Álgebra.
}

Assim, o mesmo pode ser dito sobre as proporções, que podem ser observadas nos livros didáticos de Aritmética, como, por exemplo, Silva (1923) e Souza (1910), e em livros de Álgebra como o de Euler (1828), considerado uma obra de referência.

\section{A RELAÇÃO DO ENSINO DE EQUIDIFFERENÇA E PROPORÇÃO COM A ÁLGEBRA}

Como já foi apontado anteriormente, é possível observar o uso de expressões como "termo desconhecido" ou "incógnita" na abordagem de equidifferença ou proporção em livros didáticos de Aritmética. Isto pode ressaltar uma relação deste ensino com o ensino de Álgebra da instrução secundária, haja vista que a determinação de uma incógnita geralmente está ligada ao processo de resolução de equações. A presença dos dois tipos de

primitivas e originais de que uma entidade ê constituída [...]" e, com isso, a “[...] noção de elementos tem como consequiêncía que existe uma seriação lógica e contínua de todas as proposições de uma dada ciência às quais todos os elementos podem ser integrados muito naturalmente" (SCHUBRING, 2003, p. 63). Assim, estes livros trazem consigo um "verdadeiro conjunto básico das proposições-germes" (SCHUBRING, 2003, p. 68) que se constituem como os elementos de uma ciência. 
proporções no livro Elementos de Álgebra de Euler e a relação com os conteúdos de progressão que, aparentemente, não faziam parte do ensino elementar ajudam a fortalecer ainda mais esta inquietação.

Antes de continuar a discussão aqui pretendida, é importante apresentar a nomenclatura sobre os termos de uma proporção.

Figura 1 - Nomenclatura empregada aos termos de uma proporção.

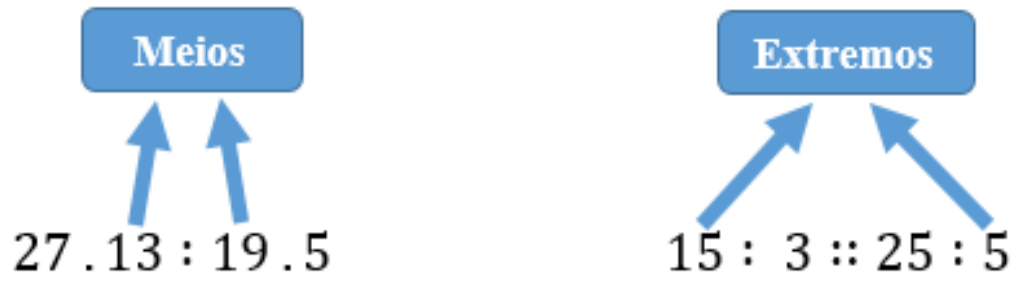

Fonte: elaborado pelos autores.

Deste modo, termos são considerados como de mesma espécie quando ambos são "meios" ou "extremos" de uma proporção. Semelhantemente, se um termo é de uma espécie, os "outros termos" são os da espécie a qual ele não pertence, por exemplo, se um termo é um "meio" então os "outros termos" são os "extremos" da proporção.

A partir disso Silva (1923) apresenta duas regras: regra 1 - "Numa equidifferença a somma dos meios é egual a somma dos extremos” (p. 40); regra 2 - "Numa proporção o producto dos meios é egual ao producto dos extremos" (p. 41). Em outros termos, dada a equidifferença $a . b:: c$. $d$ e a proporção $e: f::$ $g: h$, a regra de Silva (1923) indica que $b+c=a+d$ e que $f \times g=e \times h^{14}$.

A partir desta regra, Trajano (1922) apresenta o procedimento para a

\footnotetext{
${ }^{14}$ Estas duas expressões advêm do fato de que: a equidifferença a.b::c.d implica que $a-b=c-$ $d$, no que somando $b$ e $d$ aos dois lados da igualdade se obtém $a-b+b+d=c-d+d+b$, ou seja, $a+d=c+b$; já na proporção e:f::g:h, tem-se que $e: f=g: h$, disto basta multiplicar ambos lados da igualdade por $f$ e $h$ que se obtém $(e \times f \times h): f=(g \times f \times h): h$, que é igual a $e \times h=g \times f$.
} 
resolução da questão 9:3 :: 18: $x$, em que o produto dos extremos é $9 x^{15} \mathrm{e}$ dos meios é $3 \times 18$, no que se tem que $9 x=3 \times 18$. Segundo o autor "Como o producto dos dois meios é igual ao producto dos dois extremos, dividindo o producto dos meios por um extremo, teremos o outro extremo" (TRAJANO, 1922, p. 100), o que significa dizer que ao dividir $3 \times 18=54$ por9 irá se obter o valor de $x$, ou seja, $x=54: 9=6$. Trajano (1922, p. 100) continua dizendo que "Escrevendo-se os tres termos [...] e fazendo-se o cancellamento, obtem-se mais rapidamente o mesmo resultado", isto é, escrever $x=\frac{3 \times 18}{9}$, ao invés da regra de Silva (1923), toma menos tempo no processo de resolução.

Trajano (1922) usa, de forma implícita, a ideia de operações inversas uma vez que a passagem da expressão $3 \times 18=9 x$ para a igualdade $\frac{3 \times 18}{9}=x$ leva em consideração a divisão dos dois lados da igualdade por 9. Este processo pode ser observado explicitamente no livro de Leyssenne (1911) na Figura 2.

Figura 2 - Resolução de uma proporção com um termo desconhecido.

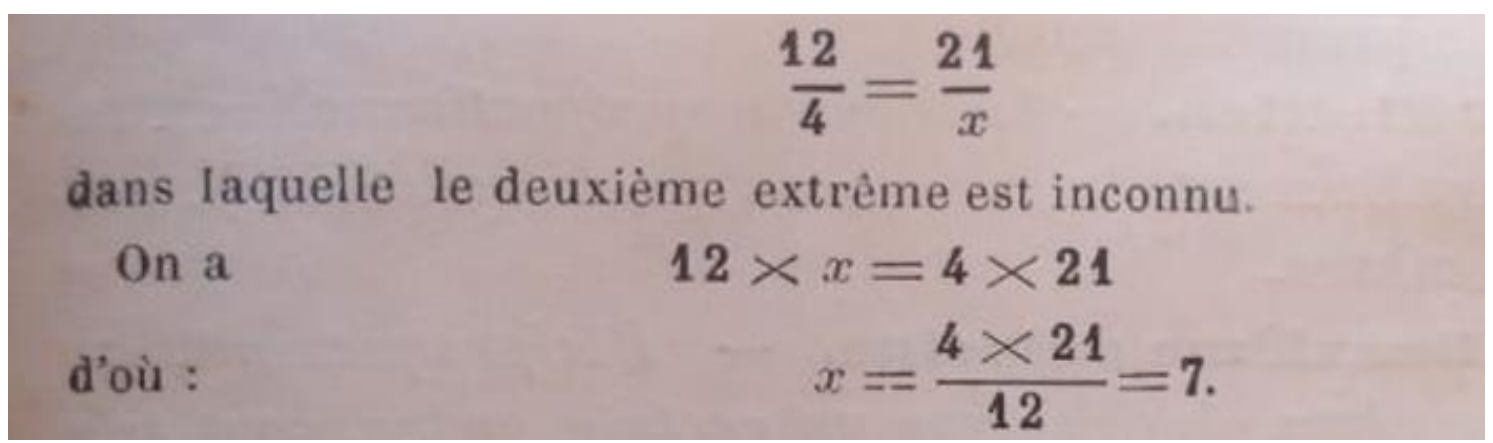

Fonte: Leyssenne (1911, p. 313).

A resolução de uma equidifferença ou proporção, quando um dos quatro termos é desconhecido, proposta por Silva (1923) se dá a partir da ideia de que "O termo desconhecido de uma equidifferença é egual á somma dos outros dois menos o termo conhecido de mesma especie" (SILVA, 1923, p. 40) e que "O termo incognito de uma proporção discontinua é egual ao produto dos

15Para evitar confusão, o produto $9 \times x$ será denotado aqui como $9 x$. 
outros dois dividido pelo termo conhecido da mesma especie" (SILVA, 1923, p. 41). Assim, abordagem de Silva (1923) é um pouco mais direta, uma vez que não perpassa a ideia de operações inversas. Nesta perspectiva, a resolução da proporção 9: $3:: 18: x$, sob a ótica do autor, se dá pela forma: como $x$ é um extremo, o produto "dos outros dois" é $3 \times 18$ e assim $x=\frac{3 \times 18}{9}$, no que não se observa a divisão pelo número 9 como resultado de uma divisão por 9 dos dois lados da igualdade

Ao contrário de Silva (1923), Leyssenne (1911) primeiro traz um exemplo para depois apresentar esta última regra. Tais enunciados advêm das regras 1 e 2 expostas por Silva, a partir do uso de operações inversas, mas isto não é apresentado em seu livro. A Figura 3 mostra um exemplo de resolução de uma proporção segundo a regra apresentada por Silva (1923) e à qual, Trajano (1922) indica chegar mais rápido ao resultado.

Figura 3 - Exemplo da determinação do termo desconhecido em uma proporção.

Exemplo. $-12: 4:: x: 5 ; x=\frac{12 \times 5}{4} ; 15$ é a quarta proporcional de 12,4 e 5.

Fonte: elaborado ${ }^{16}$ a partir de Silva (1923, p. 41).

A obra de Leyssenne (1911) e a de Silva (1923) contribuem também para mostrar que as abordagens deste conteúdo não são únicas, em que algumas, como a de Silva, tendem a um enfoque mais prático, um viés aritmético, e outras, como a de Leyssenne, em uma perspectiva voltada à apresentação de saberes algébricos, como se observa na Figura 2. O livro de Souza (1910) demonstra que um ponto de vista semelhante ao de Leyssenne também pode ser observado nos livros didáticos brasileiros. A Figura 4 mostra a abordagem de Souza (1910), na qual é possível observar que o autor enuncia os procedimentos

${ }^{16} \mathrm{O}$ arquivo que se teve acesso à obra de Silva (1923) fornecia imagens de baixa qualidade, de modo que se optou por refazer as figuras retiradas do texto seguindo exatamente a forma apresentada pelo autor. 
algébricos, vinculados às operações inversas, para se determinar o valor de $\mathrm{x}$.

Figura 4 - Exemplo da determinação de um termo desconhecido em uma proporção na qual se destacam os procedimentos algébricos.

Seja a proporção $24: 8:: x: 9$

Vamos provar que 0 termo desconhecido $x=\frac{24 \times 9}{8}$

Pela propriedade fundamental temos $8 \times x=24 \times 9$.

Um igualdade não se altera quando so divide ambos os membros pelo mesmo numero, por isso vamos dividir ambos os membros desta igualdade por 8 que é o termo que está multiplicando a incognita $x$ e assim teremos :

$$
\frac{8 \times x}{8}=\frac{24 \times 9}{8}
$$

Porém, no primeiro membro, uós temos 8 que multiplica e 8 que divide, simplifica-se, isto $\dot{e}$, o quociente sendo a unidade, corta-se este numero do dividendo e divisor o que da :

$$
\begin{aligned}
& \quad \frac{8 \times x}{8}=\frac{34 \times 9}{8} \\
& \text { ou } x=\frac{24 \times 9}{8} \text { como se queria provar. }
\end{aligned}
$$

Fonte: Souza (1910, p. 152).

De maneira similar, ao se resolver a equidifferença $12.4: 15 . x$, deve-se relembrar que esta é equivalente à expressão $12-4=15-x$ e que a resolução de tal equidifferença é, então, o mesmo que resolver uma equação do primeiro grau. Contudo, utilizando a regra apresentada por Silva (1923) a solução é obtida fazendo $x=4+15-12=7$, sem que se passe pela notação da equação do primeiro grau ou utilize saberes como operações inversas na resolução da questão.

Outras regras vinculadas ao conteúdo também são apresentadas. Silva (1923, p. 40) indica que "Se, numa equidifferença [..], o termo incognito for o 
repetido, elle será egual a semi-somma dos outros dois e receberá a denominação de media arithmetica”. O autor logo segue com um exemplo, apresentado na Figura 5 .

Figura 5 - Exemplo da determinação do termo desconhecido em uma proporção.

Exemplos. -12. $x: x .4 ; x=\frac{12+4}{2}=8 ; 8$ é a media arithmetica de 12 e 4 .

Fonte: elaborado a partir de Silva (1923, p. 41).

Se a "somma dos meios é egual a somma dos extremos" (SILVA, 1923, p. 40) for aplicada ao exemplo da figura anterior, obtêm-se que $x+x=12+4$ ou ainda $2 x=12+4$. Novamente, pode-se observar que o autor propõe a resolução de uma equação do primeiro grau que faria uso da operação inversa de dividir ambos os lados da igualdade por 2, mas sem que isto seja explicitado ao leitor.

Uma segunda regra é apresentada por Silva (1923, p. 41): "Se, numa proporção continua, o termo incognito for o repetido, ele será egual á raiz quadrada do producto dos outros dois. Receberá o nome media proporcional”. Ao que o autor exibe o exemplo $x: 4:: 9: x$ e que a solução seria $x=\sqrt{4 \times 9}=$ $\sqrt{36}=6$. Leyssenne (1911) expõe a mesma regra, mas antes expõe o seguinte exemplo:

Figura 6 - Exemplo da determinação de um termo desconhecido repetido em uma proporção. 


\section{Soit la proportion :}

$$
\frac{9}{x}=\frac{x}{4}
$$

dans laquelle $x$ est moyenne proportionnelle entre 9 et 4 .

On a :

d’où :

$$
x^{2}=9 \times 4
$$

$$
x=\sqrt{9 \times 4}=6 .
$$

Fonte: Leyssenne (1911, p. 314).

No exemplo de Leyssenne (1911), que na notação de proporção é 9: $x$ :: $x: 4$, pode-se verificar, mais uma vez, que o autor utiliza propriedades algébricas para obter a segunda equação a partir da regra que indica que o produto dos meios é igual ao produto dos extremos, dado que o produto de $x$ e $x$ seria igual a9 $\times 4$ e, com isto, que $x^{2}=9 \times 4$. Após obter a equação do segundo grau, para chegar à terceira equação o autor faz uso da operação inversa do quadrado de um número, que é a raiz quadrada, sem, no entanto, enunciar este procedimento. Novamente é possível perceber que Leyssenne (1911) e Silva (1923) apresentam a mesma regra, mas sob abordagens distintas, sendo que o primeiro busca mostrar o processo que leva à regra, assim destacando saberes algébricos no decorrer da resolução, e o segundo prezando para uma explanação mais prática do processo, focando mais nos aspectos aritméticos do que algébricos. A abordagem de Souza (1910) mescla a abordagem utilizada pelos dois autores, primeiro a resolução prática se assemelha à perspectiva aritmética e, em seguida, a explicação desse desenvolvimento, destacando os saberes vinculados ao procedimento, aproximando-se então de uma apresentação algébrica. 
Figura 7 - Exemplo da determinação de um termo desconhecido repetido em uma proporção na qual se destacam os procedimentos algébricos.

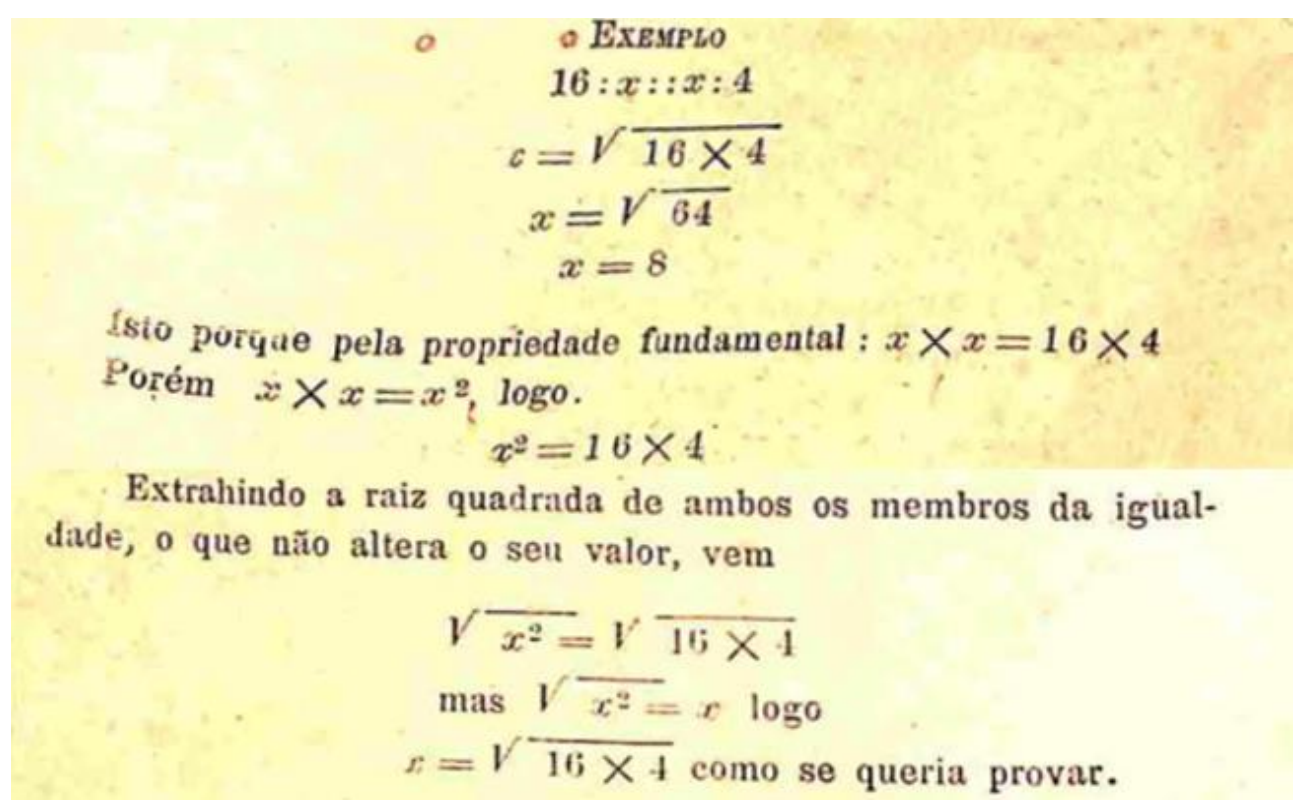

Fonte: Souza (1910, p. 153-154).

Uma abordagem que mais se aproxima da Álgebra pode ser observada no livro de L. L. (1916), que tem como foco a formação do professor do ensino elementar. Para mostrar o enunciado "Em toda equidifferença, um meio é egual á somma dos extremos menos o outro meio”, o autor (L. L., 1916, p. 89) aponta que a equidifferença $x-a=b-d$ poderia ser reescrita, segundo a propriedade que diz que a soma dos meios é igual à soma dos extremos, na forma $x+d=$ $b+a$ e que ao se subtrair $d$ de ambos os lados da equação seria obtida a igualdade $x=b+a-d$. Neste processo, é possível notar o movimento de transformação de uma regra enunciada, para determinar o termo desconhecido da equidifferença, em uma fórmula, sem a utilização de números e fazendo uso da propriedade algébrica da subtração de ambos os lados da igualdade na busca por demonstrar a regra.

Nesse sentido, outros conteúdos que poderiam ser ligados ao ensino de Álgebra, por fazerem uso de saberes algébricos, também são observados em livros de Aritmética para o ensino elementar. Um exemplo disso é o conteúdo de regra de três, no qual Silva (1923) apresenta a solução através do mesmo 
método utilizado na resolução de proporções. Com isso, a determinação de um valor desconhecido fica atrelada à utilização de uma fórmula e não aos saberes algébricos, como é possível observar na Figura 8.

Figura 8 - Exemplo de regra de três simples.

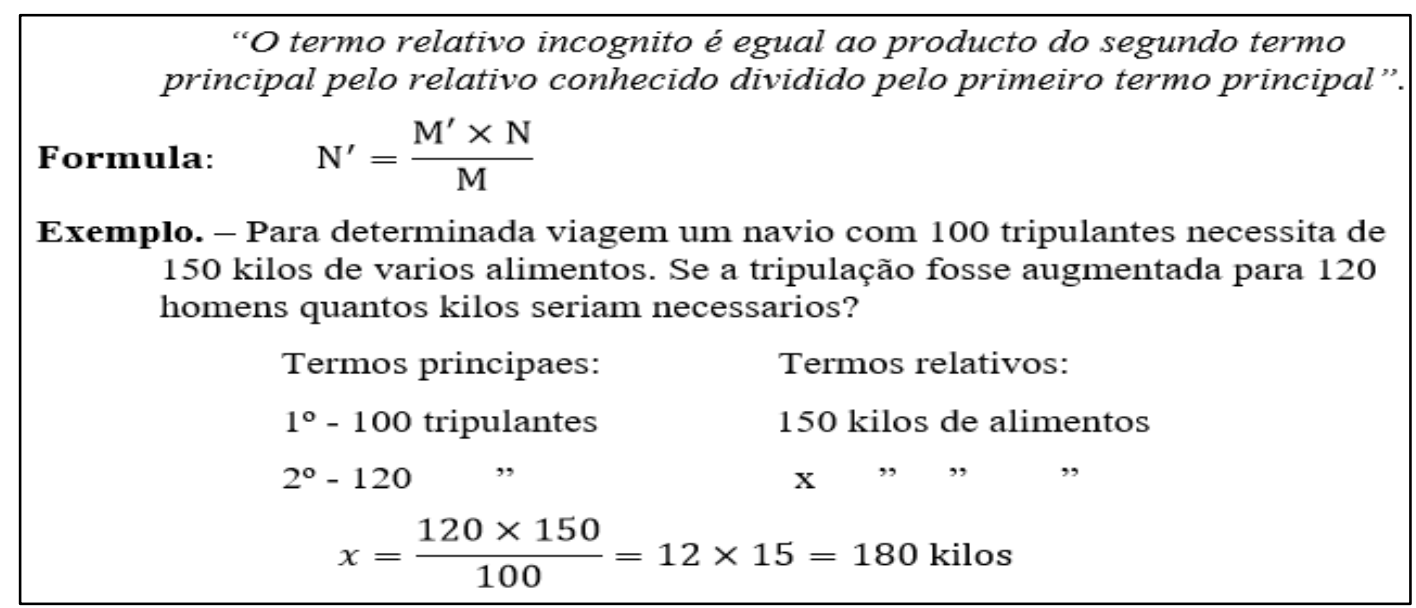

Fonte: elaborado a partir de Silva (1923, p. 42).

Nesta perspectiva, contudo, não são apresentadas abordagens usualmente associadas ao ensino de equações, como a ideia da igualdade a partir do "equilíbrio de uma balança", que leva a entender que uma operação pode ser realizada de um lado da igualdade desde que também seja efetuada no outro. O livro de Smith (1904), ao propor uma Álgebra para o ensino elementar, traz essa discussão ao questionar o que aconteceria, em uma balança em equilíbrio, "se os multiplicarmos os pesos pelo mesmo número? Dividi-los pelo mesmo número? Adicionarmos pesos iguais a ambos os lados? Subtrair pesos iguais nos dois lados?” (p. 4). Desta forma, o método empregado se mostra apropriado para a disciplina Aritmética, ou seja, saberes algébricos abordados sob uma perspectiva da Aritmética, para a solução implícita de equações pelo uso de fórmulas estipuladas e não com base em saberes algébricos. 


\section{EQUIDIFFERENÇA E PROPORÇÃO COMO UMA PREPARAÇÃO PARA O ENSINO DE ÁLGEBRA?}

Segundo Rodriguês e Costa (2019), no final do século XIX um movimento pela reestruturação do ensino secundário estadunidense tem início. O movimento leva à constituição da Comissão dos dez, que deveria estudar e propor mudanças para a melhoria da instrução secundária do país. Dentre os apontamentos feitos pela comissão, foi indicado que a reestruturação do ensino secundário necessitaria que também se reformulasse o ensino elementar do país. Nesta reorganização da instrução elementar, a Álgebra deveria ser instituída nos dois últimos anos deste ensino (NEA, 1894).

Com objetivo semelhante ao da Comissão dos dez, uma Comissão dos quinze é então formada para propor mudanças para o ensino elementar estadunidense (RODRIGUÊS; COSTA, 2019). O relatório da Comissão dos quinze foi subdividido em três partes, sendo que as discussões em relação aos conteúdos e disciplinas do ensino são apresentadas no Relatório da subcomissão de correlação de estudos na educação elementar (HARRIS et al., 1895). Bem como a Comissão dos dez apontou, a Comissão dos quinze também chega à conclusão de que o ensino de Álgebra deveria ser instituído nos dois últimos anos da instrução elementar, de forma a auxiliar no processo de resolução de problemas complexos da Aritmética e como uma introdução à Álgebra do ensino secundário.

O primeiro registro que aponta a chegada destas discussões ao Brasil, em 1918, dá-se com Othello de Souza Reis, que realiza uma conferência intitulada Os dois ultimos annos de arithmetica, na escola primaria, segundo a Comissão dos quinze (REIS, 1918a; REIS, 1918b). Nesta conferência, Reis (1918a, 1918b) mostra principalmente quais seriam as visões da comissão acerca da aplicação dos conteúdos algébricos para a resolução de problemas de Aritmética e como estes poderiam aliviar processos que, sem o uso de saberes algébricos, seriam longos e exaustivos. Além disso, a conferência de Reis, bem como sua publicação na revista A Escola Primaria e, posteriormente, sua 
publicação no prefácio do livro de Reis (1919) Algebra - Primeiros Passos, leva a concluir que estas discussões também eram necessárias no âmbito brasileiro.

No que se refere ao ensino de Álgebra para a instrução elementar, segundo a Comissão dos quinze (HARRIS et al., 1895)

\begin{abstract}
No sétimo ano do curso elementar deveriam ser ensinados equações do primeiro grau e a solução de problemas de aritmética que recaem sobre o tema de proporções ou da chamada "regra de três," junto com outros problemas contendo soluções complexas [...]. No oitavo ano equações do segundo grau poderiam ser aprendidas, e outros problemas avançados de aritmética resolvidos de maneira mais satisfatória do que por métodos numéricos (p. 245-246, tradução pelos autores).
\end{abstract}

Há uma grande relação entre o que é estipulado para o ensino elementar de Álgebra segundo a comissão e o que se observou, implicitamente, na abordagem de equidifferença e proporção na Aritmética, uma vez que equações do primeiro e segundo graus surgem como proposta para este nível de ensino. Na citação anterior, do relatório da comissão (HARRIS et al., 1895), pode-se ainda perceber que proporções e regra de três fazem parte de possíveis aplicações da Álgebra do ensino elementar na Aritmética. Estas duas observações ressaltam que mesmo com a presença do ensino de equidifferença e proporção, na Aritmética, foi necessário propor a instituição de um ensino de Álgebra para a instrução elementar.

Nesse sentido, é possível concluir que mesmo que no ensino de proporções exista a possibilidade da abordagem de saberes algébricos, sendo o professor, talvez, o principal responsável pelo nível de explicitação desses saberes aos estudantes, isto ainda não se constituiria como uma preparação para a Álgebra do ensino secundário. Deste modo, um ensino introdutório para a Álgebra secundarista seria necessário, uma vez que o ensino de equidifferença e proporção devesse assumir uma perspectiva mais próxima da Aritmética do que da Álgebra, voltada à praticidade do uso de fórmulas na determinação de 
valores desconhecidos e aplicação de operações.

\section{CONCLUSÃO}

Ao se debruçar sobre o ensino de equidifferença e proporção, buscou-se compreender como este ensino se relacionava com outros conteúdos presentes na instrução. Inicialmente, foi possível estabelecer uma relação com progressões, aritméticas e geométricas, de modo que a abordagem de proporções pode se caracterizar como introdutória para o estudo de progressões.

Além disso, um olhar mais aprofundado nos livros didáticos possibilitou destacar que uma relação também poderia ser estabelecida com saberes algébricos. Na busca por determinar o valor de uma incógnita, ou um valor desconhecido, em uma equidifferença ou proporção, as resoluções acabam por perpassar conteúdos como equações do primeiro grau e segundo grau. Contudo, não é toda abordagem que permite a observação explicita desses temas no processo de resolução, uma vez que o foco, aparentemente, desse ensino estava em uma perspectiva Aritmética, voltada à aplicação de fórmulas e das quatro operações básicas na resolução de problemas.

Ainda assim, é possível observar que, historicamente, mesmo no ensino de Aritmética, pode-se encontrar a presença do ensino de saberes algébricos. Estes elementos destacam que os conteúdos de equidifferença e proporção, bem como as progressões, transitam entre um ensino de Álgebra e Aritmética. Por estarem presentes em livros das duas áreas, são as escolhas de abordagens dos autores que podem determinar uma maior aproximação com uma área ou outra.

O movimento observado nos Estados Unidos para a introdução do ensino de uma introdução à Álgebra na instrução elementar chegaria anos depois nas discussões acerca do ensino brasileiro. As propostas ressaltam a importância da abordagem dos conteúdos de equações do primeiro e segundo 
graus para simplificar a resolução de problemas complexos da Aritmética, mas também para uma preparação para a Álgebra do ensino secundário. A necessidade dos saberes algébricos na instrução elementar levanta dois pontos relevantes quanto ao ensino de proporções: em primeiro lugar, que as abordagens de equidifferença e proporção não levavam à discussão dos saberes algébricos implícitos na resolução de seus problemas, sendo necessária uma discussão explícita destes no ensino de Álgebra; em segundo lugar, que a abordagem implícita desses saberes algébricos na Aritmética não seria(m) suficiente(s) para a introdução ou preparação do estudante para o ensino de Álgebra na instrução secundária.

Contudo, há ainda outros temas importantes que podem ser tratados em pesquisas acerca desta temática, como a inserção do ensino de proporções na Aritmética da instrução elementar, ou ainda, a análise de livros de Álgebra do ensino secundário na busca em saber como as proporções aqui apresentadas se relacionam com esta Álgebra.

\section{REFERÊNCIAS}

BRASIL. Base Nacional Comum Curricular. 2018

BRASIL. Parâmetros curriculares nacionais. 1997.

BARBARESCO, Cleber Schaefer. Saberes a ensinar aritmética na Escola de Aprendizes Artífices de Santa Catarina (1909-1937) lidos nos documentos normativos e livros didáticos. 2019. 183f. Dissertação (Mestrado em Educação Científica e Tecnológica). Universidade Federal de Santa Catarina, Programa de Pós-Graduação em Educação Científica e Tecnológica, Florianópolis, 2019.

CABRITA, Francisco. A algebra no ensino primario. A Escola Primária, ano 1, n. 12 , p. $360-361,1917 \mathrm{~b}$.

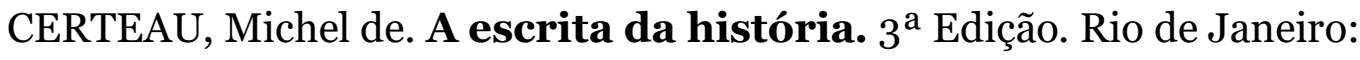

Forense Universitária, 2013, p. 45-111. 
CHERVEL, André. História das disciplinas escolares: reflexões sobre um campo de pesquisa. Teoria \& Educação, n. 2, p. 177-229, 1990.

CHOPPIN, Alain. História dos livros didáticos e das edições didáticas: sobre o estado da arte. Educação e Pesquisa, v. 30, n. 3, p. 549-566, 2004.

COJORI, Florian. Origin of the names arithmetical and geometrical progression and proportion. SCHOOL SCIENCE AND MATHEMATICS, v. 22, n. 8, p. 734-737, 1922.

EULER, Leonard. Elements of Algebra. 4. ed. London: Longman, Rees, Orme, And Co., 1828.

HARRIS, William T.; GREENWOOD, James M.; GILBERT, Charles B.; JONES, Lewis H.; MAXWELL, William H. Report of The Sub-Committee On The Correlation Of Studies. Elementary Education. Educational Review, vol. 9, p. 230-303, 1895 .

L.L. Elementos de arithmetica. Campinas: Typografia da Casa Ideal, 1916.

LE GOFF, Jacques. História e Memória. Tradução de Bernardo Leitão. et al. Campinas, SP. Editora da UNICAMP, 1990.

LEYSSENNE, Pierre. Traité D’Arithmétique: Théorique et Pratique. Librairie Armand Colin, Paris, 1911.

NEA. Report of the committee of ten on secondary school studies. Nova York: American Book Company, 1894.

NEVES, Thais Duarte; SOARES, Flávia dos Santos. Progressões Aritmética e Geométrica: visões a partir dos livros de Aritmética e Álgebra de Serrasqueiro e Ottoni. Revemop, Ouro Preto, MG, v. 1, n. 2, p. 313-316, maio/ago. 2019.

OLIVEIRA, Marcus Adenilson. Antonio Bandeira Trajano e a renovação pedagógica lida em livros escolares: ensinar aritmética de modo intuitivo (final do século xix). Revista História da Educação, 23, 1-41, 2019.

REIS, Othello de Souza. ALGEBRA - PRIMEIROS PASSOS. Livraria Drummond, Rio de Janeiro, 1919.

REIS, Othello de Souza. Os dois ultimos annos de arithmetica, na escola primaria, segundo a Comissão dos quinze. A Escola Primaria. Rio de Janeiro, ano 3, n. 1, p. 11-15, 1918a.

REIS, Othello de Souza. Os dois ultimos annos de arithmetica, na escola primaria, segundo a Comissão dos quinze (continuação). A Escola Primaria. 
Rio de Janeiro, ano 3, n. 2-3, p. 41-43, 1918b.

RODRIGUÊS, Jeremias Stein; COSTA, David Antonio. A Comissão dos Quinze e os Primeiros Movimentos Acerca do Ensino da Álgebra na Escola Primária Brasileira. Acta Scientiae, v. 21, n. 6, p. 150-172, 2019.

SHUBRING, Gert. Análise histórica de livros de matemática: Notas de aula. Tradução de Maria Laura Magalhães Gomes. Campinas, SP: Autores Associados. 2003.

SHUBRING, Gert. Pesquisar sobre a história do ensino da Matemática: metodologia, abordagens e perspectivas. In: ENCONTRO DE INVESTIGAÇÃO EM EDUCAÇÃO MATEMÁTICA, XII, Beja. Actas..., Beja: SPCE (Sociedade Portuguesa de Ciências da Educação), 2004, p. 5-20.

SILVA, Ruy de Lima e. Arithmetica Pratica e Formulario. Rio de Janeiro: Besnard Frère, 1923.

SMITH, David Eugene. Grammar School Algebra. Boston: Ginn \& Company, 1904.

SOUZA, Antonio Monteiro de. Arithmetica Elementar. 4. ed. Rio de Janeiro: Rodrigues \& C, 1910.

TRAJANO, Antonio. Arithmetica Elementar Illustrada. $92^{\mathrm{a}}$ ed. Rio de Janeiro: Livraria Francisco Alves, 1922.

JEREMIAS STEIN RODRIGUÊS é professor do Instituto Federal de Santa Catarina - IFSC. Doutorando do Programa de Pós-Graduação em Educação Científica e Tecnológica da Universidade Federal de Santa Caratina - UFSC.

E-mail: jeremias.stein@ifsc.edu.br

(1) http://orcid.org/0000-0002-7869-5856

DAVID ANTONIO DA COSTA é doutor em Educação Matemática pela Pontifícia Universidade Católica de São Paulo. Docente no Centro de Ciências da Educação e no Programa de Pós-Graduação em Educação Científica e Tecnológica da Universidade Federal de Santa Catarina. 
E-mail: david.costa@ufsc.br

(D) http://orcid.org/0000-0003-4493-9207

Recebido em: 25 de abril de 2020

Aprovado em: 01 de julho de 2020

Editora responsável: Dóris Almeida 